\title{
Two-stage contests with preferences over style
}

\author{
Todd R. Kaplan ${ }^{1,2}$ (1) $\cdot$ David Wettstein $^{3}$
}

Received: 6 September 2016 / Accepted: 24 August 2021

(c) The Author(s) 2021

\begin{abstract}
Many grant applications have a preliminary stage where only a select group are invited to submit a full application. Similarly, procurement contracts by governments are often awarded through a two-stage procedure. We model and analyze such environments where the designer cares about the style of the application as well as its quality. The designer has the option of choosing an initial stage, where contestants can enter and learn about their desirability while the designer learns about their style. We determine closed form solutions for equilibrium outcomes and designer payoffs and use this to analyze whether or not a second stage is desirable, different rules for deciding who will advance, and whether or not to communicate the number of contestants that qualify for the second stage.
\end{abstract}

Keywords Contests · Innovation · All-pay auctions $\cdot$ Mechanism design

JEL Classification C70 · D44 · L12 · O32

We wish to thank participants from the Current Frontiers in the Theory of Contests workshop at the Max Planck Institute for Tax Law and Public Finance, Munich, 2016, as well as helpful comments by a co-editor and two referees. We would like to dedicate this paper to Dr.Michael Feige (Ben-Gurion University) and the other victims in the 2016 terror attack at the Sarona Market, Tel Aviv, as well as the workers in the cafes there. During that period and in that locale, we derived the bulk of our results..

$凶$ Todd R. Kaplan

toddrkaplan@gmail.com

David Wettstein

wettstn@bgu.ac.il

1 Department of Economics, University of Exeter, Exeter EX44PU, UK

2 Department of Economics, University of Haifa, 31905 Haifa, Israel

3 Department of Economics, Ben-Gurion University of the Negev, 84105 Beer-Sheva, Israel 


\section{Introduction}

There are many examples of contests run in two stages. Often grant applications have a preliminary stage where only some of the applications advance to the second stage. The Leverhulme Foundation has several funding schemes that require two stages (for instance for a research project grant): an outline application and a detailed application. ${ }^{1}$ In 2012, there were 908 applications to this scheme approximately 50\% made it to the second round and $40 \%$ of those received funding $(20 \%$ of the original received funding). This two-stage process is common for large grants of several UK funding agencies (NERC, ESRC, etc.) as well as used in the establishment of centers for research excellence (I-CORE) in Israel. In its 'Second Wave' of funding starting in 2013, the latter had 26 out of 67 applications advance to the second stage of which 12 were chosen for funding.

In architecture, it is common to hold a contest for determining a building design. One of the earliest examples was a contest for the design to rebuild the Houses of Parliament in 1836 after a fire. A recent prestigious example of such a contest is when the Mumbai City Museum ran a design competition for a $\$ 45$ million additional wing (using Malcolm Reading Consultants to run the competition). Expressions of interest were received from 104 architects worldwide with 8 teams shortlisted. The jury, which consisted of 11 distinguished members, chose New York-based Steven Holl as the winner. There are also a plethora of smaller architecture contests using two stages. $^{2}$

We also note that such a practice is common in advertising. There is a call for a request for a proposal (RFP) sent to half a dozen ad agencies that asks not only background questions and who will be on the core team, but creative questions about approach. After seeing the RFP responses, the top two or three are invited to present the ideas (perhaps after feedback). ${ }^{3}$ Other examples include government procurement, talent show contests, and television series pilots.

There are two characteristics about these contests that are worth noting: (1) This practice appears to be most common in areas where a particular preference or style might be a major factor in selecting the winner. (2) It appears that sometimes (and sometimes not) the proposed number of finalists is announced.

We find that (1) may be because the contestants are not aware of the preferences of the designer, who may favor some contestants over others. This can be thought of as the economics grant committee either preferring theoretical research over empirical research or vice-versa. The reason for (2) is less obvious and may simply be a feature of the optimal contest design.

\footnotetext{
1 The applications to the two stages do not differ in the general idea: there cannot be substantial differences in the intentions, aims, objectives, personnel or budget between the two applications.

2 The Garden Museum in South London sought an architect to take forward plans to extend the museum in a second phase of renovation. In a two-stage contest, the value of the contract to the architects was estimated between $£ 380,000$ and $£ 420,000$ and expressions of interest were due by January 20,2013 . It was announced that up to five practices would be shortlisted for the job. In another advertisement, the Tricycle Theatre in Kilburn, North London sought an architect for its $£ 2.4$ million refurbishment. There was no mention of how many would be shortlisted. See http://www.bdonline.co.uk/home/competitions for other examples.

3 From personal correspondence with Rachel Greene, a public relations and media relations consultant.
} 
We suppose that style is an exogenous feature of a proposal, but quality is a function of effort. For simplicity, we assume that the designer's preference over style is dichotomous: either preferred or not. We also assume for simplicity that the first stage is limited in scope such that a contestant can either put in the effort required for the designer to determine his or her type or not. This matches many real world contests where the first stage is meant to weed out those with an inappropriate style. It may not be feasible to put in extra effort or at least have that extra effort visible. Such may be the case in a two-stage grant proposal where the first stage proposal is limited to a 1000 words.

We model and analyze such environments where the designer wants to maximize the best overall effort (as opposed to the total effort of the contestants) by a preferred style. In designing the contest, the designer may choose between a one-stage contest and a two-stage contest. The advantage of the two-stage contest is that the designer learns the type of a contestant if that player puts forth some minimal effort. The disadvantage is that this minimal effort does not contribute to the efforts in the second stage. If the designer chooses a two-stage contest, the designer also has other options. He may choose to advance only a specific number of contestants (two) that satisfy his preferences or anyone that satisfies his preferences. ${ }^{4} \mathrm{He}$ may also choose whether or not to announce how many made it to the second stage.

Here we use a framework where information is symmetric among contestants and there is complete information about the value of winning the contest (see Baye et al. 1996, and more recently Kaplan et al. 2003; Siegel 2009). Recently there have been a number of papers on multi-stage contests (see Cohen et al. 2018; Sela 2011, 2012, 2017; Segev and Sela 2014a, b as well as experiments comparing one-stage to twostage (see Sheremeta 2010). There has also been research where the designer has preferences over style (see Kaplan 2012). Also related to our paper is research on entry in contests where there is potentially an unknown number of entrants (see Fu et al. 2011, 2014, 2015; Chen et al. 2017) as well as auctions with a unknown number of bidders (see McAfee and McMillan 1987). Troncoso-Valverde (2018) studies information revelation in markets in which auctioneers running second-price auctions compete for buyers and provides sufficient conditions for the existence of an equilibrium where information is revealed. The contribution of our paper is adding the possibility of a two-stage design to a contest where the designer cares about style as well as quality and can reveal information about the number of contestants.

The paper proceeds as follows. In the next section, we present the model, followed by the equilibrium analysis in Sect. 3. We rank the possible contest designs in terms of number of stages, qualification criteria, and revealed information in Sect. 4 . We discuss the robustness of our results in Sect. 5 and conclude in Sect. 6.

\section{The contest environment}

There are $N$ contestants competing for a prize of value $V$. Style is exogenous and each contestant independently has a probability $p \in(0,1)$ of having a style that the

\footnotetext{
4 We study these extremes since they are the most prominent to study and appropriate if results are monotonic in the number of contestants above two.
} 
designer desires and $(1-p)$ of not having a desired style. The contest can be run in one or two stages. Contestants decide how much effort to exert in each stage. The designer of the contest cares only about the highest effort exerted in the last stage by a contestant of a style that he desires. The designer is only able to determine the style of a contestant if that contestant's effort is $m$ or above. This makes intuitive sense (one has to write at least a short proposal). Furthermore, awarding the prize to a contestant with a non-desired style is prohibitively costly.

As a benchmark, we study a one-stage contest with a minimum effort of $m$ and compare this benchmark with several two-stage contest designs. In all the two-stage contests, the first stage requires that contestants put in effort $m$ in order to have the possibility of advancing. Thus, each contestant doing so has his/her style revealed by the end of stage one. We note that putting in more effort than $m$ does not increase one's chances of advancing. Thus, the first stage is really about screening contestants. We assume that $V$ is large enough to ensure that, in the equilibria analyzed in the various designs, all contestants will choose to enter in the first stage.

The two-stage contests differ along three aspects: whether there is minimal effort required in the second stage, the criteria to qualify for the second stage, and information revealed to qualifying contestants. The minimal effort in the second stage can be $m$ (a $2 m$ environment) or zero (an $m$ environment). (Note that we use this nomenclature since doing so counts the aggregate minimal effort needed to participate in the second stage.) Whether it is a $2 m$ or $m$ environment may at times not be a choice of the designer, but an exogenous feature of the environment. ${ }^{5}$ The designer can choose between two qualifying rules: (1) all those that are discovered to have a desired style advance (all pass), or (2) of the contestants eligible to move to the second stage, two randomly advance (if there are indeed two) (random two). Finally, after the first stage but before second stage decisions are made, the designer can choose to inform or not the contestants about the number of contestants advancing to the second stage (inform or not inform).

\section{Equilibrium analysis}

In this section, we derive the equilibrium strategies and outcomes for several possible contest designs. We start with the benchmark case of a one-stage contest and proceed to analyze several families of two-stage contests.

\subsection{Benchmark case: one stage}

Here we examine the equilibrium where all contestants choose effort according to a distribution function $F$. We note that unlike Baye et al. (1996), there is a unique equilibrium (in particular, there are no asymmetric equilibria). See Appendix A.

\footnotetext{
${ }^{5}$ Having a minimum of 0 in the second stage means contestants can just resubmit what they did before, that is, copy and paste. Having a minimum of $m$ in the second stage means the second stage could be more elaborate requiring some effort to have a comprehensible proposal. The first logical point to start is that writing a comprehensible proposal would require the same amount of effort as writing a comprehensible proposal in the first stage (equal to $m$ ). For instance, in the case of auditions, practicing lines for the part.
} 
In order to ensure entry by all contestants, $V$ must satisfy, $p(1-p)^{N-1} V>m .^{6}$ This constraint guarantees that a contestant that enters and bids $m$ (which would be in the support of the equilibrium) would make positive profit. When bidding $m$, the contestant would only win if he is the only contestant with a desired style, which happens with probability $(1-p)^{N-1} p$.

For $F$ to be part of an equilibrium, it must satisfy:

$$
p[p F(x)+(1-p)]^{N-1} V-x=(1-p)^{N-1} p V-m .
$$

The RHS of (1) is the expected profit of putting in effort $m$. The LHS of (1) is the expected profit of putting in $x \geq m$. The probability of having the preferred style is $p$ and, given this, the probability of winning is that each other contestant either does not have the preferred style (with probability $1-p$ ) or has the preferred style but puts in less effort (with probability $p F(x)$ ).

Solving (1) for $F(x)$ yields:

$$
\begin{aligned}
F(x) & =\frac{\left[\frac{x-m}{p V}+(1-p)^{N-1}\right]^{\frac{1}{N-1}}-(1-p)}{p} \\
& =\left[\left(\frac{1-p}{p}\right)^{N-1}+\frac{x-m}{p^{N} V}\right]^{\frac{1}{N-1}}-\frac{(1-p)}{p}
\end{aligned}
$$

with support $\left[m, p V\left[1-(1-p)^{N-1}\right]+m\right]$.

To determine the designer's one-stage profits, denoted by $\Pi^{\text {one }}$, we proceed to evaluate the expected value of the highest effort put forth by a contestant with a preferred style. We define a distribution $G(x)$ by $G(x)=p F(x)+(1-p)$. The function $G$ represents the cumulative distribution of preferred effort by an individual contestant given that we don't know whether or not the contestant has a preferred type. Hence, we replace the case where effort is not from a preferred type by an atom of size $(1-p)$ at zero. Now $\Pi^{\text {one }}=\int_{m}^{\bar{x}} x d G^{N}$ where $G(x)=\left[(1-p)^{N-1}+\frac{x-m}{p V}\right]^{\frac{1}{N-1}}$ and $\bar{x}=p V\left[1-(1-p)^{N-1}\right]+m$. Hence, $\Pi^{\text {one }}=m+\frac{N p V}{2 N-1}+\frac{(N-1) p V(1-p)^{2 N-1}}{2 N-1}-$ $(1-p)^{N-1}(m(1-p)+p V)$.

Next, we consider the first of several two-stage contests.

\subsection{Two stages: all pass}

With two stages and all pass (denoted by AP in mathematical expressions), all the contestants that put forward effort $m$ in stage one and have the preferred style pass to the second stage. A contestant that makes it to the second stage learns that he has the preferred style. Also, depending upon the information condition, the contestant may or may not know how many other contestants also have a preferred style. In the latter

\footnotetext{
6 This full entry condition (and ones that follow in the paper) is sufficient but not necessary since one can have full entry in the first stage with equality, but equality does not ensure full entry.
} 
case since all that have the preferred style make it to the second stage, making it to the second stage does not affect a contestant's estimate about how many other contestants with a preferred style are competing in the second stage.

\subsubsection{All pass: $2 \mathrm{~m}$, not inform}

For full participation in stage one, we require

$$
p(1-p)^{N-1} V>m(1+p) .
$$

The LHS of (2) is the expected payoff of participating, given by the expected probability of being alone in the second stage times the prize. The RHS of (2) is the expected cost of participating, given by the minimum effort $m$ needed in stage one and the probability of advancing to stage two and again putting in the minimum effort.

Again we look for a symmetric equilibrium with a distribution function $F$ that represents effort in the second stage. As in the one stage case, this is again the unique equilibrium. ${ }^{7}$ For $F$ to be part of an equilibrium, the corresponding $G$ distribution function must satisfy:

$$
G(x)^{N-1} V-x=(1-p)^{N-1} V-m .
$$

The RHS of (3) is the expected profit of putting in effort $m$ in which case winning occurs with probability of $(1-p)^{N-1}$. The LHS of (3) is the expected profit of putting in effort $x \geq m$. Note that as opposed to (1), here at the second stage, each contestant already knows he has the preferred style. Thus, moving from one stage to two stages effectively increases the prize from $p V$ to $V$.

Solving (3) for $F(x)$ yields (by first solving for $G(x)$ ):

$$
\begin{aligned}
F(x) & =\frac{\left[\frac{(1-p)^{N-1} V+x-m}{V}\right]^{\frac{1}{N-1}}-(1-p)}{p} \\
& =\left[\left(\frac{1-p}{p}\right)^{N-1}+\frac{x-m}{p^{N-1} V}\right]^{\frac{1}{N-1}}-\frac{(1-p)}{p}
\end{aligned}
$$

with support $\left[m, V\left[1-(1-p)^{N-1}\right]+m\right]$.

Proceeding similarly to the one-stage environment, we can calculate two-stage profits, when contestants are not informed and must pay at least $m$ in the second stage, denoted by $\Pi_{2 m, N I}^{A P}$. We obtain $\Pi_{2 m, N I}^{A P}=\int_{m}^{\bar{x}} x d G^{N}$ where $G(x)=$ $\left[(1-p)^{N-1}+\frac{x-m}{V}\right]^{\frac{1}{N-1}}$ and $\bar{x}=V\left[1-(1-p)^{N-1}\right]+m$. Hence, $\Pi_{2 m, N I}^{A P}=$ $m-m(1-p)^{N}+\frac{N V}{2 N-1}+(1-p)^{N-2} V\left[\frac{(N-1)(1-p)^{N+1}}{2 N-1}-(1-p)\right]$.

\footnotetext{
7 The second stage equilibrium is unique for the same reasons as the one stage equilibrium is. Solving the game backwards yields the unique overall equilibrium.
} 


\subsubsection{All pass: $m$, not inform}

This environment is identical to the previous one except for the fact there is no minimal bid $m$ required in the second stage. Hence, the expected cost of participating is now just $m$. Thus, for full participation in stage one, we now require

$$
p(1-p)^{N-1} V>m .
$$

Again we look for a symmetric equilibrium with a distribution function $F$ that represents effort in the second stage. As in the previous two cases, there is a unique equilibrium. Looking at the second stage, for $F$ to be part of an equilibrium, the corresponding $G$ distribution function must satisfy:

$$
G(x)^{N-1} V-x=(1-p)^{N-1} V .
$$

The RHS of (5) differs from the RHS of (3) in that $m$ need not be expended in the second stage. The LHS of (5) is identical to the LHS of (3).

Solving (5) for $F(x)$ yields:

$$
\begin{aligned}
F(x) & =\frac{\left[\frac{(1-p)^{N-1} V+x}{V}\right]^{\frac{1}{N-1}}-(1-p)}{p} \\
& =\left[\left(\frac{1-p}{p}\right)^{N-1}+\frac{x}{p^{N-1} V}\right]^{\frac{1}{N-1}}-\frac{(1-p)}{p}
\end{aligned}
$$

with support $\left[0, V\left(1-(1-p)^{N-1}\right)\right]$.

To determine the designer's profits, denoted by $\Pi_{m, N I}^{A P}$, we proceed similarly to before, to obtain $\Pi_{m, N I}^{A P}=\int_{0}^{\bar{x}} x d G^{N}$ where now $G(x)=\left[(1-p)^{N-1}+\frac{x}{V}\right]^{\frac{1}{N-1}}$ and $\bar{x}=V\left(1-(1-p)^{N-1}\right)$. Hence $\Pi_{m, N I}^{A P}=\frac{(N-1)(1-p)^{2 N-1}-(2 N-1)(1-p)^{N-1}+N}{2 N-1} V$.

\subsubsection{All pass: $2 \mathrm{~m}$, inform}

For full participation in stage one, we now require

$$
p(1-p)^{N-1}(V-m)>m .
$$

The LHS of (6) is the expected payoff to contestant $i$ after entering the contest - the payoff in the second stage is strictly positive only when one contestant qualifies (and equals $V-m$ ), the probability of which is $p(1-p)^{N-1}$, while the RHS is the cost of entering.

With probability $N p(1-p)^{N-1}$ only one contestant will participate in the second stage. Since the contestant knows this, the designer will get $m$. For $i \geq 2$, there will be $i$ contestants in the second stage with probability $\left(\begin{array}{c}N \\ i\end{array}\right) p^{i}(1-p)^{N-i}$, and the symmetric 
equilibrium $F(x)$ must satisfy $F(x)^{i-1} V-x=0$ for all $x \geq m$. (Note as in Baye et al. (1996) and similar in form, there are additional asymmetric equilibria when three or more contestants make it to the second stage. For consistency when comparing to when there is a unique equilibrium, we focus on the symmetric equilibrium.) Hence, each contestant bids according to the distribution function:

$$
F_{i}(x)=\left\{\begin{array}{l}
\left(\frac{x}{V}\right)^{\frac{1}{i-1}} \text { if } x \geq m, \\
\left(\frac{m}{V}\right)^{\frac{1}{i-1}} \quad x<m .
\end{array}\right.
$$

The designer's profits, denoted by $\Pi_{2 m, I}^{A P}$, are then given by $\Pi_{2 m, I}^{A P}=\sum_{i=2}^{N}\left(\begin{array}{c}N \\ i\end{array}\right)$ $p^{i}(1-p)^{N-i} \int_{m}^{V} x d\left(\frac{x}{V}\right)^{\frac{i}{i-1}}+N \cdot p(1-p)^{N-1} m=\sum_{i=2}^{N}\left(\begin{array}{c}N \\ i\end{array}\right) p^{i}(1-p)^{N-i} \frac{i}{2 i-1}(V-$ $\left.m\left(\frac{m}{V}\right)^{\frac{i}{i-1}}\right)+N \cdot p(1-p)^{N-1} m$.

\subsubsection{All pass: $m$, inform}

For full participation in stage one, we require $p(1-p)^{N-1} V>m$.

With probability $N p(1-p)^{N-1}$ only one contestant will participate in the second stage. Since the contestant knows this, the designer will get 0 . With probability $\left(\begin{array}{c}N \\ i\end{array}\right) p^{i}(1-p)^{N-i}$, there will be $i \geq 2$ contestants in the second stage, the symmetric equilibrium distribution function $F$ must then satisfy $F(x)^{i-1} V-x=0$. As in all pass $2 m$ inform, when there are three or more contestants, there are additional asymmetric equilibria.

Hence, in equilibrium each contestant bids according to the distribution function:

$$
F_{i}(x)=\left(\frac{x}{V}\right)^{\frac{1}{i-1}} \text { on the interval }[0, V] .
$$

This leads to the following payoff to the designer, denoted by $\Pi_{m, I}^{A P}: \Pi_{m, I}^{A P}=$ $\sum_{i=2}^{N}\left(\begin{array}{c}N \\ i\end{array}\right) p^{i}(1-p)^{N-i} \int_{0}^{V} x d\left(\frac{x}{V}\right)^{\frac{i}{i-1}}=V \sum_{i=2}^{N}\left(\begin{array}{c}N \\ i\end{array}\right) p^{i}(1-p)^{N-i} \frac{i}{2 i-1}$.

\subsection{Two stages: random two pass}

We now look at where the designer randomly chooses two contestants among those that have the preferred style. With random two pass (denoted by ran2 in mathematical expressions), a contestant advancing to the second stage learns something about the other contestants. The fact that a contestant was selected means that he is more likely to be the only one with the preferred style (by Bayes' rule).

Consider for example the case where $N=3$ and $p=0.5$. If a contestant has a preferred style, then there is a $25 \%$ chance he is the only one with a preferred style and $50 \%$ chance that there is exactly one other contestant with a preferred style and a $25 \%$ chance that all three contestants have a preferred style. Hence, in all pass, if a contestant makes it to the second stage, the probability that he is the only one that advanced is $25 \%$. In random two, if a contestant has a preferred style, he advances 
with a 2/3 probability when there are two others with a preferred style, otherwise, he would always advance. Thus, the probability of being the only one that advanced given that one advanced is $\frac{25}{25+50+\frac{2}{3} \cdot 25} \approx 27 \%$.

\subsubsection{Random two: $2 \mathrm{~m}$, not inform}

Denote the probability that a contestant advances to the second stage by

$$
P_{a}=p\left[(1-p)^{N-1}+\sum_{i=1}^{N-1}\left(\begin{array}{c}
N-1 \\
i
\end{array}\right) p^{i}(1-p)^{N-1-i} \frac{2}{i+1}\right]
$$

Denote the probability that given a contestant advances, there are no other contestants that advanced by $p_{a}=\frac{p(1-p)^{N-1}}{P_{a}}=\frac{N p}{\frac{2}{(1-p)^{N-1}}-N p-2(1-p)}$ (using the same logic as above for $N=3)$. For full participation in stage one, we require $P_{a}\left(p_{a} V-m\right)>m$.

In equilibrium, in stage $2, F(x)$ satisfies:

$$
\left[\left(1-p_{a}\right) F(x)+p_{a}\right] V-x=p_{a} V-m .
$$

Solving for $F(x)$ yields:

$$
F(x)=\frac{x-m}{\left(1-p_{a}\right) V} .
$$

Note the equilibrium is unique since only one or two contestants advance to the second stage. The designer's payoff, denoted by $\Pi_{2 m, N I}^{\mathrm{ran} 2}$, is then:

$$
\begin{aligned}
\Pi_{2 m, N I}^{r a n 2}= & N p(1-p)^{N-1} \int_{m}^{\bar{x}} x d F+\left(1-(1-p)^{N}-N p(1-p)^{N-1}\right) \int_{m}^{\bar{x}} x d F^{2} \\
= & N p(1-p)^{N-1}\left(m+\frac{1}{2} V\left(1-p_{a}\right)\right)+\left(1-(1-p)^{N}-N p(1-p)^{N-1}\right) \\
& \times\left(m+\frac{2}{3} V\left(1-p_{a}\right)\right) .
\end{aligned}
$$

\subsubsection{Random two: $m$, not inform}

For full participation in stage one, we require $P_{a}\left(p_{a} V-m\right)>0$. In equilibrium, in stage 2, $F(x)$ satisfies:

$$
\left[\left(1-p_{a}\right) F(x)+p_{a}\right] V-x=p_{a} V .
$$

Solving for $F(x)$ yields: $F(x)=\frac{x}{\left(1-p_{a}\right) V}$. The equilibrium is also unique here and the designer's payoff, denoted by $\Pi_{m, N I}^{\operatorname{ran2}}$, is then given by $\Pi_{m, N I}^{\operatorname{ran2}}=N p(1-$ $p)^{N-1} \int_{0}^{\bar{x}} x d F+\left(1-(1-p)^{N}-N p(1-p)^{N-1}\right) \int_{0}^{\bar{x}} x d F^{2}=N p(1-p)^{N-1}\left(\frac{1}{2} V(1-\right.$ $\left.\left.p_{a}\right)\right)+\left(1-(1-p)^{N}-N p(1-p)^{N-1}\right)\left(\frac{2}{3} V\left(1-p_{a}\right)\right)$. 


\subsubsection{Random two: $2 \mathrm{~m}$, inform}

For full participation in stage one, we require $p(1-p)^{N-1}(V-m)>m$. If only one contestant participates in the second stage, then the contestant knows this and the designer will get $m$ (which is the minimum effort). If there are two contestants in the second stage and this is commonly known, the unique equilibrium distribution function $F(x)$ must satisfy $F(x) V-x=0$ for all $x \geq m$. Thus, the overall expected profits, denoted by $\Pi_{2 m, I}^{r a n 2}$, are given by $\Pi_{2 m, I}^{r a n 2}=N p(1-p)^{N-1} m+\left(1-(1-p)^{N}-N p(1-\right.$ $\left.p)^{N-1}\right) \int_{m}^{V} x d F^{2}=N p(1-p)^{N-1} m+\left(1-(1-p)^{N}-N p(1-p)^{N-1}\right)\left(\frac{2 V}{3}-\frac{2 m^{3}}{3 V^{2}}\right)$.

\subsubsection{Random two: $m$, inform}

For full participation in stage one, we require $p(1-p)^{N-1} V>m$. If only one contestant participates in the second stage, then when in the inform design the contestant knows he is the only contestant that advanced and the designer will get 0 . When there are two contestants in the second stage, the unique equilibrium distribution function $F(x)$ must satisfy $F(x) V-x=0$ for all $V \geq x \geq 0$. The designer's expected profits, denoted by $\Pi_{m, I}^{r a n 2}$, are then $\Pi_{m, I}^{r a n 2}=\left(1-(1-p)^{N}-N p(1-p)^{N-1}\right) \int_{0}^{V} x d F^{2}=$ $\left(1-(1-p)^{N}-N p(1-p)^{N-1}\right)\left(\frac{2 V}{3}\right)$.

\section{Ranking the designs}

We now proceed to compare the various designs from the point of view of the designer. The designer is interested in the expected highest effort by a contestant with a preferred style. If there are two stages, then it is the expected highest effort in the second stage only. We refer to this as designer profit. We start by comparing the two qualification rules.

\subsection{Random two pass versus all pass}

Proposition 1 For $N>2$, in any of the four two-stage designs, random two pass generates higher designer profit than all pass, that is, $\Pi_{2 m, N I}^{r a n 2}>\Pi_{2 m, N I}^{A P}, \Pi_{m, N I}^{r a n 2}>$ $\Pi_{m, N I}^{A P}, \Pi_{2 m, I}^{r a n 2}>\Pi_{2 m, I}^{A P}$ and $\Pi_{m, I}^{r a n 2}>\Pi_{m, I}^{A P}$.

Proof The latter two inequalities can be shown to hold by directly looking at the differences: $\Pi_{2 m, I}^{r a n 2}-\Pi_{2 m, I}^{A P}=\left(1-(1-p)^{N}-N p(1-p)^{N-1}\right)\left(\frac{2 V}{3}-\frac{2 m^{3}}{3 V^{2}}\right)-$ $\left[\sum_{i=2}^{N}\left(\begin{array}{c}N \\ i\end{array}\right) p^{i}(1-p)^{N-i} \frac{i}{2 i-1}\left(V-m\left(\frac{m}{V}\right)^{\frac{i}{i-1}}\right)\right]>\left(1-(1-p)^{N}-N p(1-p)^{N-1}\right)\left(\frac{2 V}{3}-\right.$ $\left.\frac{2 m^{3}}{3 V^{2}}\right)-\left[\sum_{i=2}^{N}\left(\begin{array}{c}N \\ i\end{array}\right) p^{i}(1-p)^{N-i} \frac{2}{3}\left(V-m\left(\frac{m}{V}\right)^{2}\right)\right]=0$

and

$\Pi_{m, I}^{r a n 2}-\Pi_{m, I}^{A P}=\left(1-(1-p)^{N}-N p(1-p)^{N-1}\right)\left(\frac{2 V}{3}\right)-V \sum_{i=2}^{N}\left(\begin{array}{c}N \\ i\end{array}\right) p^{i}(1-$ p) $)^{N-i} \frac{i}{2 i-1}>$ 


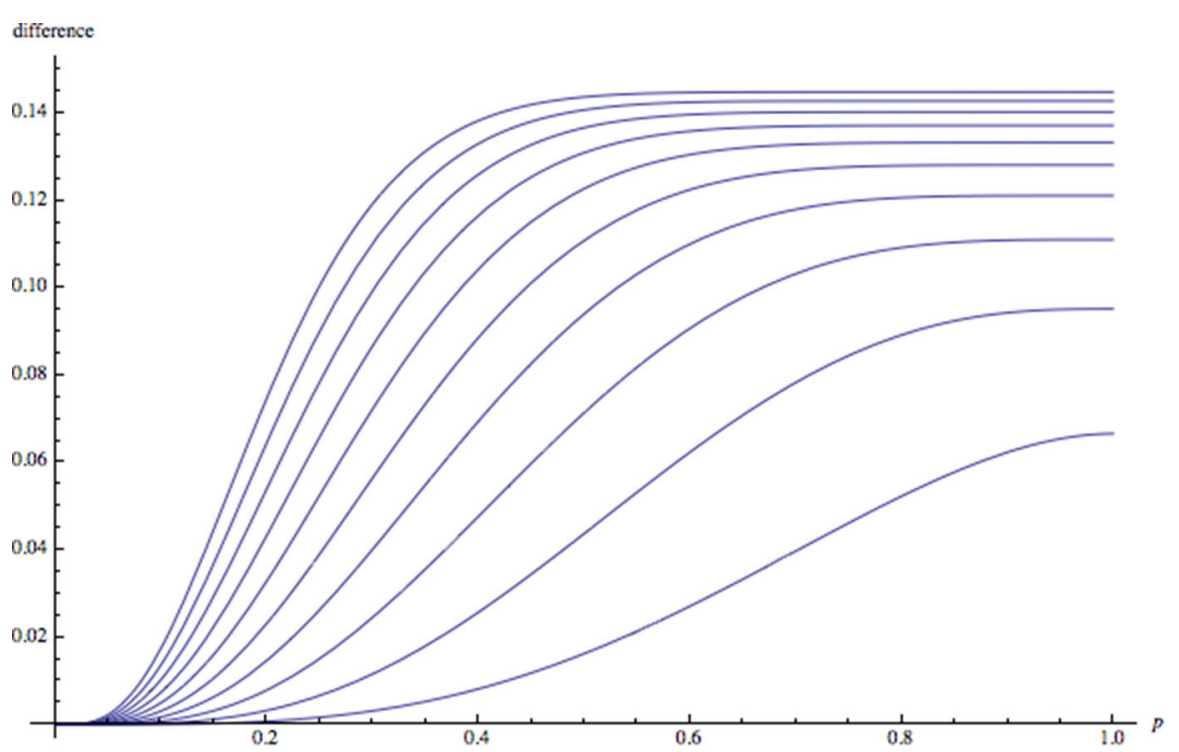

Fig. 1 Difference of $\Pi_{2 m, N I}^{\text {ran2 }}-\Pi_{2 m, N I}^{A P}$ (divided by $V$ ) versus $p$ when $n$ varies from 3 to 12 . Higher curve corresponds to higher $n$

$$
\left(1-(1-p)^{N}-N p(1-p)^{N-1}\right)\left(\frac{2 V}{3}\right)-V \sum_{i=2}^{N}\left(\begin{array}{c}
N \\
i
\end{array}\right) p^{i}(1-p)^{N-i} \frac{2}{3}=0 .
$$

Intuition is that with all pass the profit is strictly lower when three or more contestants have a preferred style. We now show the first two inequalities hold for the case of $N=3$. The difference for the $2 m$ case is $\Pi_{2 m, N I}^{r a n 2}-\Pi_{2 m, N I}^{A P}=$ $V\left[\frac{2 p^{4}\left(3 p^{3}-15 p^{2}+26 p-15\right)}{15\left(p^{2}-3\right)}\right]$. At $p=1 / 2$, this is strictly positive. There are real roots at 0 and 1.28. Thus, the difference for $2 m$ is strictly positive for any $p \in(0,1)$. Likewise, the difference for the $m$ case is $\Pi_{m, N I}^{r a n 2}-\Pi_{m, N I}^{A P}=V\left[\frac{p^{2}\left(3 p^{3}-15 p^{2}+26 p-15\right)}{15\left(p^{2}-3\right)}\right]$. At $p=1 / 2$, this is strictly positive. There are real roots at 0 and 1.58. Again, this shows that the difference for $m$ is strictly positive for any $p \in(0,1)$.

For $N>3$, the exercise is similar. By plotting the difference for both cases in Figs. 1 and 2, we see that the difference is increasing in $N$ and hence always positive. ${ }^{8}$

Our results for the informed case mirror the results in Serena (2017) and Kaplan et al. 2003, who both examine models where the designer's objective is maximizing the highest effort (as opposed to the total effort). Serena (2021) shows in a Tullock contest that limiting the number of contestants to two is optimal. Likewise, Kaplan et al. (2003), show in an all-pay auction with complete information, it is also optimal limiting the number of participants to two. However, a new result implied by Proposition 1 is that when the contestants are not informed, it is optimal to reduce the expected number of contestants in the second stage to strictly less than two (by moving from all pass to random two and this may be from above two. For instance, when $N=3$ and $p=0.8$,

$\overline{8 \text { We have also done this up to } N}=200$. 


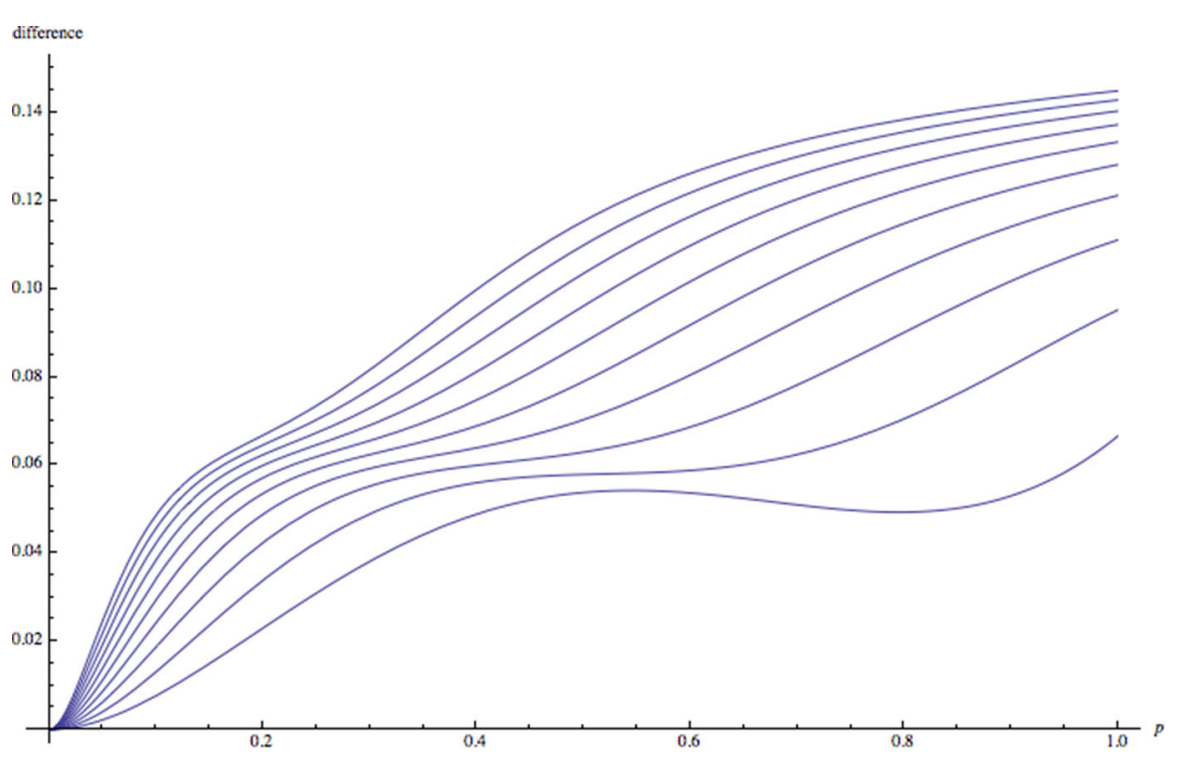

Fig. 2 Difference of $\Pi_{m, N I}^{r a n 2}-\Pi_{m, N I}^{A P}$ (divided by $V$ ) versus $p$ when $n$ varies from 3 to 12 . Higher curve corresponds to higher $n$

with all pass, the expected number in the second stage is 2.4 while with random two pass, the expected number is 1.888 . This even holds when expected number in all pass is precisely 2 (as when $p=2 / 3$ ).

The results from Proposition 1 support the use of random two rather than all pass, yet we do see instances of the all pass design. This might be due to considerations outside the scope of our model such as motivations other than pure effort maximization: First, when just two contestants pass, there may be a cooperation between the two, leading to reduced payoff for the designer. Second, there might be public outcry against an arbitrary decision, due to concerns regarding possible discrimination and favoritism on part of the designer. Next we compare the desirability of requiring a minimum effort in the second stage as well.

\subsection{Minimum effort $m$ versus $2 m$}

Here we find that in the case where contestants are not informed, it is always better to have a minimum effort in both stages. This contrasts to when the contestants are informed where it is only worthwhile for small $p$. Intuitively, for uninformed the uncertainty regarding the number of competitors has a dampening effect on the desire to put forth an effort unless it is likely to be the highest, since efforts are sunk. This effect induces the competitors to put in efforts also between 0 and $m$ when there is no minimum effort requirement. A minimum effort requirement in stage 2 shifts the support of equilibrium efforts upwards. (For large enough $V$, contestants are not deterred from participating by the minimum effort requirements.)

These two findings are proven formally in the following two propositions. 
Proposition 2 In all pass and not inform, a minimum bid in both stages yields higher designer profit than a minimum bid in just one stage, that is, $\Pi_{m, N I}^{A P}<\Pi_{2 m, N I}^{A P}$.

Proof Note that $\Pi_{2 m, N I}^{A P}=\int_{m}^{V\left[1-(1-p)^{N-1}\right]+m} x d\left[(1-p)^{N-1}+\frac{x-m}{V}\right]^{\frac{N}{N-1}}$ and $\Pi_{m, N I}^{A P}=\int_{0}^{V\left[1-(1-p)^{N-1}\right]} x d\left[(1-p)^{N-1}+\frac{x}{V}\right]^{\frac{N}{N-1}}$. We perform a change of variables $z=x-m$ to obtain

$$
\begin{aligned}
\Pi_{2 m, N I}^{A P} & =\int_{0}^{V\left[1-(1-p)^{N-1}\right]}(z+m) d\left[(1-p)^{N-1}+\frac{z}{V}\right]^{\frac{N}{N-1}} \\
& =\Pi_{m, N I}^{A P}+\int_{0}^{V\left[1-(1-p)^{N-1}\right]} m d\left[(1-p)^{N-1}+\frac{z}{V}\right]^{\frac{N}{N-1}}>\Pi_{m, N I}^{A P} .
\end{aligned}
$$

We now make the comparison for the case of random two.

Proposition 3 In random two pass and not inform, a minimum bid in both stages yields higher designer profit than a minimum bid in just one stage, that is, $\Pi_{m, N I}^{\mathrm{ran} 2}<\Pi_{2 m, N I}^{\mathrm{ran} 2}$.

Proof The difference of profits is $\Pi_{2 m, N I}^{r a n 2}-\Pi_{m, N I}^{r a n 2}=\left(1-(1-p)^{N}\right) m>0$.

In the case where contestants are informed regarding the number of contestants who qualified, the ranking depends on $p$. We see this in the next two propositions.

Proposition 4 In both random two pass and all pass when informed, (i) designer profit with a minimum bid in one stage is smaller than with a minimum bid in two stages when the chance of having a desired style is sufficiently small, that is, $\Pi_{m, I}^{r a n 2}<\Pi_{2 m, I}^{r a n 2}$ and $\Pi_{m, I}^{A P}<\Pi_{2 m, I}^{A P}$ if $p$ is close to 0 (and small enough $m$ to ensure entry); (ii) and the opposite holds when the chance of having a desired style is sufficiently large, that is, $\Pi_{m, I}^{r a n 2}>\Pi_{2 m, I}^{r a n 2}$ and $\Pi_{m, I}^{A P}>\Pi_{2 m, I}^{A P}$ if $p$ is close to 1 (and small enough $m$ to ensure entry).

Proof Considering first the random two pass, note that $\Pi_{2 m, I}^{r a n 2}-\Pi_{m, I}^{r a n 2}=N p(1-$ $p)^{N-1} m-\left(1-(1-p)^{N}-N p(1-p)^{N-1}\right)\left(\frac{2 m^{3}}{3 V^{2}}\right)$. When $p=1$, this expression is negative and, when $p=0$, the expression is 0 . The derivative of the expression w.r.t. $p$ at 0 equals $N m$, which is strictly positive.

Considering the all pass, we have $\Pi_{2 m, I}^{A P}-\Pi_{m, I}^{A P}=-\sum_{i=2}^{N}\left(\begin{array}{c}N \\ i\end{array}\right) p^{i}(1-p)^{N-i} \frac{i}{2 i-1}$ $\left(m\left(\frac{m}{V}\right)^{\frac{i}{i-1}}\right)+N \cdot p(1-p)^{N-1} m$. Now similarly to random two pass, when $p=1$, this expression is negative and, when $p=0$, the expression is 0 . The derivative of the difference w.r.t. $p$ at 0 equals $N m$, which is strictly positive. We note that when $p$ is close to 0 or $p$ is close to 1 , the range of $m$ that allows entry in both stages converges to 0 as well. However, such cases are feasible since one can construct examples by fixing $p$ and choosing $m$ accordingly to guarantee entry. 
The intuition for Proposition 4 is that when informed that there is only one contestant that advanced, that contestant would put effort at the minimum bid (or zero if there is no minimum bid). When $p$ is close to 0 , a contestant that advances is most likely to be alone, thus, in this case it is better to require a minimum effort in the second stage. If informed that more than one contestant has advanced, the second-stage contestants would put a positive mass on exerting zero effort when a minimum bid is required. This would not happen without a minimum bid. When $p$ is close to 1 , there is likely to be more than one contestant, thus, in this case it is better to refrain from imposing a minimum bid in the second stage.

We remark that it is plausible that in some types of contests the designer cannot choose between the two environments of $m$ and $2 m$. For instance, the designer may prefer a $2 m$ design, but politically it would be difficult not to award a contract when there is a contestant with a suitable style and the designer knows this.

We now proceed to compare informing and not informing contestants.

\subsection{Informing or not informing}

Here we answer the question of whether or not the designer should let contestants know how many advance to the second stage. McAfee and McMillan (1987) show that with standard auctions and risk-neutrality there is no difference in revenue between informing and not informing, but with constant absolute risk-aversion, not informing is superior. As opposed to the auction literature, in our setup all the contestants pay their costs. With contests unlike auctions (where effort is only expended by the winner), there is a distinction between the objective of maximizing the highest effort and the objective of maximizing the total effort. Serena (2021) also looks at information revelation in contests but with the objective of maximizing total effort and the information is about the rival's types.

We note that a "not informing" policy must be credibly committed to, that is, no information is revealed regardless of the actual number of participants who make it to the second stage. Otherwise, simply not revealing the information, might be taken, to mean that the designer, who is now aware of the actual number of participants, is better off not disclosing it. For instance, if $N=2$, when both make it to the second stage it is worthwhile to say so. If only one makes it to the second stage, then it is better to not to reveal it (assuming that the contestants believe there is a chance that two advanced). Hence, in the absence of a credible commitment to keep the number of contestants secret, non-disclosure would unravel. We now proceed to rank the inform and not inform policies for the various scenarios and start with the all pass design.

Proposition 5 (i) With all pass, minimum bid only in the first stage, designer profit is higher not informing than informing for two contestants or a small enough chance of having a desired style, that is, $\Pi_{m, I}^{A P}<\Pi_{m, N I}^{A P}$ for $N=2$ or $N>2$ and small $p$ (and small enough $m$ to ensure entry). (ii) The opposite holds for a high chance of a desired style and three or more contestants, that is, $\Pi_{m, I}^{A P}>\Pi_{m, N I}^{A P}$ for $N>2$ and large $p$ (and small enough $m$ to ensure entry). 
Proof We note that for $N>2$, we have $\Pi_{m, I}^{A P}=\Pi_{m, N I}^{A P}$ for $p=0$ and $p=1$. Furthermore at $p=1$, the derivative of the difference $\left(\Pi_{m, N I}^{A P}-\Pi_{m, I}^{A P}\right)$ is positive and at $p=0$, the derivative of the difference is 0 with a positive second derivative. While we still must have $p \in(0,1)$ and $p(1-p)^{N-1} V \geq m$, by continuity there must exist a $p^{\prime} \in(0,1)$ such that $\Pi_{m, N I}^{A P}-\Pi_{m, I}^{A P}>0$ for all $p, 0<p \leq p^{\prime}$ and a $p^{\prime \prime} \in(0,1)$ such that $\Pi_{m, N I}^{A P}-\Pi_{m, I}^{A P}<0$ for all $p, 1>p \geq p^{\prime \prime}$. We can then find a small enough $m^{\prime}$ such that $p(1-p)^{N-1} V>m^{\prime}$ for $p=p^{\prime}$ and $p=p^{\prime \prime}$. Thus, we have a range of probabilities where the Proposition holds.

A similar result is obtained in the $2 m$ environment.

Proposition 6 (i) With all pass, minimum bid in both stages, designer profit is higher not informing than informing for two contestants or a small enough chance of having a desired style, that is, $\Pi_{2 m, I}^{A P}<\Pi_{2 m, N I}^{A P}$ for $N=2$ or $N>2$ and small $p$ and $m$. (ii) The opposite holds for $N>2$ contestants, large $p$, and a small minimum bid, that is, $\Pi_{2 m, I}^{A P}>\Pi_{2 m, N I}^{A P}$ for $N>2$, large $p$, and small enough $m$.

Proof Note that as $m$ goes to $0, \Pi_{2 m, I}^{A P} \rightarrow \Pi_{m, I}^{A P}$ and $\Pi_{2 m, N I}^{A P} \rightarrow \Pi_{m, N I}^{A P}$. Hence, the results of the previous proposition will hold here as well.

The intuition in this case is that when $p$ is almost zero, a contestant that qualifies puts in positive effort in the uninformed case since he is not certain of being the only contestant that qualified. However in the informed scenario when $p$ is almost zero with a very high probability a contestant will be informed that he is the only one and in such a case will put in zero effort. When $p$ is large a contestant that qualifies is almost certain that everyone qualified in the uninformed case and thus puts in little effort. However when informed, he learns with high probability there are less contestants than he expected (but still two or more) and will put in more effort. In other words, when $N>2$, with high likelihood, informing reduces effort when $p$ is small and increases effort when $p$ is large.

As we see in the above propositions, the ranking is ambiguous when $N>2$ in that it depends upon $p$. However, as we now see in the random two pass environment, the ranking is unequivocal in favor of not informing. We state this in the following proposition.

Proposition 7 With random two pass, designer profit is higher in not inform, that is, $\Pi_{m, N I}^{r a n 2}>\Pi_{m, I}^{r a n 2}$ and $\Pi_{2 m, N I}^{r a n 2}>\Pi_{2 m, I}^{r a n 2}$.

Proof Looking at the difference $\Pi_{m, N I}^{\text {ran2 }}-\Pi_{m, I}^{\text {ran2 }}=$

$$
\begin{gathered}
N p(1-p)^{N-1}\left(\frac{1}{2} V\left(1-p_{a}\right)\right)+\left(1-(1-p)^{N}-N p(1-p)^{N-1}\right)\left(\frac{2}{3} V\left(-p_{a}\right)\right) \\
=V \frac{N p\left(2(1-p)+(1-p)^{N}\left(1-3\left(\frac{1}{1-p}\right)^{N-1}+p(N-1)\right)\right.}{3(1-p)\left(2-2\left(\frac{1}{1-p}\right)^{N-1}+p(N-2)\right)}
\end{gathered}
$$

For $N=2$, this equals $V$ times $p^{2}(1-p) / 3$. For $N=3$, this equals $V$ times

$$
\frac{(1-p)^{2} p^{2}(3-2 p)}{3-p^{2}} \text {. }
$$




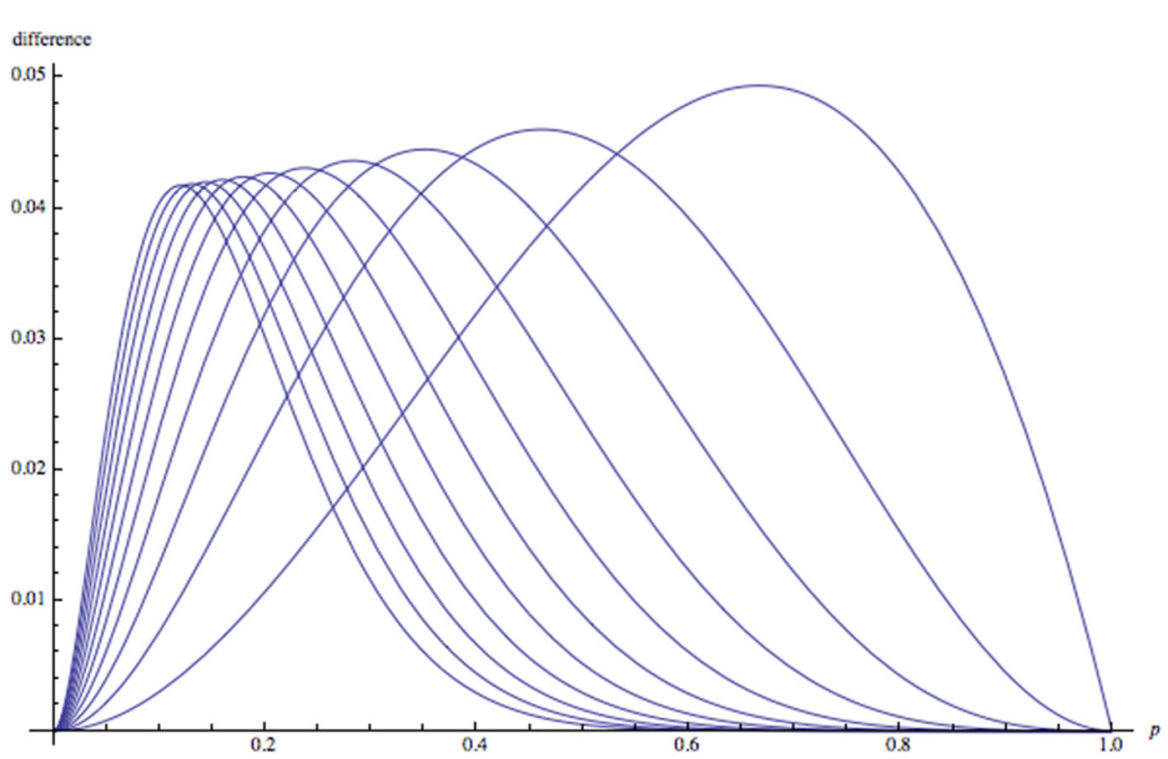

Fig. 3 Difference of $\Pi_{m, N I}^{\operatorname{ran} 2}-\Pi_{m, I}^{\operatorname{ran} 2}$ (divided by $V$ ) versus $p$ when $n$ varies from 2 to 12 . Higher $n$ corresponds to a left shift

For $N=4$, this equals $V$ times

$$
\frac{2(1-p)^{3} p^{2}\left(6-8 p+3 p^{2}\right)}{3\left(2-p^{2}(2-p)\right)}
$$

These expressions (for $N=2,3,4$ ) are strictly positive for $0<p<1$. For $N>4$, the exercise is similar. By plotting the difference in Fig. 3, we see that the difference is positive. 9

$$
\begin{aligned}
& \text { We also have } \Pi_{2 m, N I}^{\operatorname{ran} 2}-\Pi_{2 m, I}^{r a n 2}= \\
& N p(1-p)^{N-1}\left(\frac{1}{2} V\left(1-p_{a}\right)\right)+\left(1-(1-p)^{N}-N p(1-p)^{N-1}\right)\left(m-\frac{2}{3} V\left(p_{a}\right)-\frac{2 m^{3}}{3 V^{2}}\right)= \\
& \left(\Pi_{m, N I}^{r a n 2}-\Pi_{m, I}^{r a n 2}\right)+\left(1-(1-p)^{N}-N p(1-p)^{N-1}\right)\left(m-\frac{2 m^{3}}{3 V^{2}}\right)>\Pi_{m, N I}^{\text {ran2 }}-\Pi_{m, I}^{r a n 2}>0
\end{aligned}
$$
(for $0<p<1$ ).

Here the intuition for why not informing is better for the designer is that whether or not the contestants are informed they on average put in the same expected effort. However, when they are informed, relatively more of this effort is spent when there are two contestants in the second stage. Since the designer cares about the maximum effort, more effort is wasted when the contestants are informed. This is similar in logic to two contestants choosing a random effort uniform in $[0,1]$ half the time and 0 the other half of the time. It is best for the designer to have each effort used when the other contestant uses 0 effort since then on average he would have $1 / 2$ while if the efforts were put in together, the designer would have $2 / 3$ but only $1 / 2$ the time.

$\overline{9 \text { We have also done this up to } N}=200$. 


\subsection{One stage versus two stages}

We finally address the basic design question of whether or not the contest should be a one or two stage competition.

Proposition 8 With all pass, a minimum bid only in the first stage, and not inform, for small enough $m$ relative to $p$, the designer's profit is larger than in a one stage contest, that is, $\Pi_{m, N I}^{A P}>\Pi^{\text {one }}$. For large $m$, it is possible that $\Pi_{m, N I}^{A P}<\Pi^{\text {one }}$.

Proof When $m \rightarrow 0$, we have $\Pi_{m, N I}^{A P}-\Pi^{o n e}=V\left[\frac{N}{2 N-1}(1-p)+\frac{(N-1)(1-p)^{2 N}}{2 N-1}-\right.$ $\left.(1-p)^{N}\right]$. When $N=2$, this reduces to $p^{2}(3-p)(1-p) \frac{1}{3}$, which is positive between 0 and 1 . Similar to before, by plotting the differences for $N \geq 3$, we can see the relationship continues to hold. For the second part, when $N=2, m=1 / 4$, $p=1 / 2, v=1$, we find $\Pi^{\text {one }}-\Pi_{m, N I}^{A P}=\frac{1}{12}$.

The advantage of having two stages is that it avoids having the effort put in the second stage when a style isn't desirable. A contestant knowing that his style is desirable, increases the effort that he is willing to put in. The disadvantage of two stages is that if there is no minimum bid in the second stage, then efforts are lower. For small $m$, this disadvantage is negligible, but not so for larger $m$.

Proposition 9 With all pass, a minimum bid in both stages and not inform, the designer's profit is larger than in a one stage contest, that is, $\Pi_{2 m, N I}^{A P}>\Pi^{\text {one }}$.

Proof We can also show this directly by noting

$$
\Pi^{\text {one }}=\int_{0}^{p V\left[1-(1-p)^{N-1}\right]+m} x d\left[(1-p)^{N-1}+\frac{x-m}{p V}\right]^{\frac{N}{N-1}}
$$

whereas

$$
\Pi_{2,2 m, N I}^{A P}=\int_{m}^{V\left[1-(1-p)^{N-1}\right]+m} x d\left[(1-p)^{N-1}+\frac{x-m}{V}\right]^{\frac{N}{N-1}} .
$$

Since $p<1$, the inequality follows by first-order stochastic dominance.

Intuitively, when there is a minimum bid in both stages of all pass, the only difference between one stage and two stages is that a contestant with a desired style knows that he has a desired style. This effectively increases the size of the prize since without this knowledge a contestant perceives the reward of having the highest effort as $p V$ while with this knowledge the contestant knows the reward is $V$. Since the effective reward is higher, the designer's profit is larger.

Proposition 10 With all pass and inform, for small enough $m$ relative to $p$, the designer's profit is larger than in a one stage contest, that is, $\Pi_{m, I}^{A P}, \Pi_{2 m, I}^{A P}>\Pi^{\text {one }}$. For large $m$, it is possible that $\Pi_{m, I}^{A P}, \Pi_{2 m, I}^{A P}<\Pi^{\text {one }}$. 
Proof The proof follows similar lines to that of Proposition 8.

Proposition 11 The designer's profit in random two pass with minimum bids in both stages and not inform is larger than in a one stage contest, that is, $\Pi_{2 m, N I}^{r a n 2}>\Pi^{\text {one }}$. In the other cases of random two, profit is larger than the one stage with a small enough $m$ relative to $p, \Pi_{m, N I}^{r a n 2}, \Pi_{2 m, I}^{r a n 2}, \Pi_{m, I}^{r a n 2}>\Pi^{\text {one }}$. For large $m$, it is possible that profit is less, that is, $\Pi_{m, N I}^{r a n 2}, \Pi_{2 m, I}^{r a n 2}, \Pi_{m, I}^{r a n 2}<\Pi^{o n e}$.

Proof Combining Proposition 9 with Proposition 1 shows that $\Pi_{2 m, N I}^{r a n 2}>\Pi^{\text {one }}$. For the small $m$ cases, we can combine the above Propositions with Proposition 1. For large $m$, since when $N=2$, the random two case and all pass case are identical, there is also a possibility that profit is less than the one stage.

The above findings from all the sections show that $\Pi_{2 m, N I}^{r a n 2}$ is the largest profit the designer can obtain. However, to obtain this profit will require the designer to be able to commit to both not inform contestants about the number making it to the second stage as well as being able to commit to a minimum bid in the second stage.

\section{Robustness}

Cumulative efforts: In our model, effort in the first stage is not only sunk, but wasted in the sense that it does not count towards the second stage effort. Here we ask what happens if the effort is cumulative. Since a contestant does not know whether or not he has a preferred type, it is relatively cheaper to put in effort in the second stage since there is a chance of at least $(1-p)$ that the contestant would not advance. Hence, first stage efforts, should still be at the minimum. When a minimum effort is only required in the first stage, this will not affect results since the first stage effort is still sunk and everyone in stage two has already put the effort in. Likewise, if an additional minimum effort is required in the second stage (and the first stage effort does not count towards this) then again, there would be no change. Finally, in the environments where the additional minimum effort is required but the first stage effort can count towards this, then the equilibrium will be identical to our model when only a stage one minimum effort is required, that is, the $2 m$ environments with cumulative efforts will be equivalent to the $m$ environments without cumulative efforts.

Asymmetry: Allowing for asymmetry ex-ante falls outside the scope of our model, however, the qualitative results we obtain do not hinge on the symmetry assumption. For instance consider the following asymmetric environment:

Contestant 1 has prize value 6 and probability $1 / 2$ of having the desired style, contestant 2 has prize value 4 and probability $1 / 3$ of having the desired style.

In the all pass not informed scenario with $m=0$, the equilibrium distribution functions of the contestants, $F_{1}$ and $F_{2}$ for contestant 1 and 2 respectively, are given by $F_{1}(x)=F_{2}(x)=\frac{x}{2}$ with support [0,2]. The designer's payoff is given by: $\frac{1}{2}$. $\frac{2}{3} \int_{0}^{2} \frac{x}{2} d x+\frac{1}{2} \cdot \frac{1}{3} \int_{0}^{2} \frac{x}{2} d x+\frac{1}{2} \cdot \frac{1}{3} \int_{0}^{2} \frac{x^{2}}{2} d x=\frac{13}{18}$.

The first (second) term is the payoff in the case only contestant 1 (2) has the desired style, the third term is the payoff in the case both have the desired style, payoff when neither has the desired style is 0 . 
In the all pass informed scenario with $m=0$, the designer's payoff is positive only when both contestants have the desired style. In this case the equilibrium distribution functions of the contestants in the second stage after having been informed both passed are: $F_{1}(x)=\frac{x}{4}$ and $F_{2}(x)=\frac{1}{3}+\frac{x}{6}$ with support [0,4].

The designer's payoff is then given by: $\frac{1}{2} \cdot \frac{1}{3} \int_{0}^{4} x \cdot d\left(\frac{x}{4} \cdot\left(\frac{1}{3}+\frac{x}{6}\right)\right)=\frac{11}{27}$.

Hence the designer is better off not informing in this asymmetric environment as well. A full analysis of the asymmetric case is left for future work but we believe it will not invalidate the insights derived from examining the symmetric case.

\section{Discussion and conclusion}

We analyzed contest environments where the designer cares about the style and quality of the winning effort. We considered four design issues: whether to use one stage or two, requiring a minimum bid in both stages, whether to advance all qualified contestants or to place a limit, and whether or not to inform contestants about the number of contestants that advance to the second stage. These, for the most part, can be observed in actual contests run.

We found closed form solutions for the equilibrium strategies and expected designer's profits for the various contest designs. We then examined rankings between several design options. While some can be unequivocally ranked, other rankings were dependent upon the parameters defining the environment. Overall, we found that the design maximizing the highest effort is a random two design where there is no information given about the number that make it to the second stage and there is a minimum bid in each stage.

While some of our assumptions were deliberately extreme in order to allow us to solve the model, in particular with the desired style, there are cases in which desired style is really a fit or doesn't fit. This is often seen in acting where one fits the part or doesn't. ${ }^{10}$ With acting, one can also see an example where two finalists were chosen. The actors and actresses playing the children in the Brady Bunch were first narrowed down to two sets of children where two children competed for each part. The final selection was a combination of the sets. ${ }^{11}$

We also rank designs beyond the optimal since in practice we often see contests with more than two finalists. Possibly this could be due to unmodelled factors such as a concern about favoritism on part of the designer.

There are many ways to expand our work. While the purpose of this work is to focus on screening by having a two-stage mechanism, it is possible to look at parameters where the contestants' willingness to enter in the first stage is not ensured. It is also worthwhile to have contestants with heterogeneous abilities. In this case, it may be superior to allow more than two contestants to advance. Also, one can examine social welfare issues and different objective functions of the designer (such as total effort).

\footnotetext{
10 This can be where someone is needed for a body double. Also, it could be where height is a consideration. Consider casting a musical version Mary Poppins. If the director already selected an actor for Michael, the actress playing his sister Jane must be taller than that actor since Jane is supposed to be older.

11 Originally the two sets were to have matching hair color to their parent, but they dyed the hair of the actor playing Bobby in order to combine the sets.
} 
Finally, one can change the model to a scenario where the chance of having a desired style depends upon the effort put forth in stage one.

Open Access This article is licensed under a Creative Commons Attribution 4.0 International License, which permits use, sharing, adaptation, distribution and reproduction in any medium or format, as long as you give appropriate credit to the original author(s) and the source, provide a link to the Creative Commons licence, and indicate if changes were made. The images or other third party material in this article are included in the article's Creative Commons licence, unless indicated otherwise in a credit line to the material. If material is not included in the article's Creative Commons licence and your intended use is not permitted by statutory regulation or exceeds the permitted use, you will need to obtain permission directly from the copyright holder. To view a copy of this licence, visit http://creativecommons.org/licenses/by/4.0/.

\section{Appendix A: uniqueness proof for the one-stage contest}

Here we prove uniqueness of the equilibrium in the benchmark, one-stage contest. While this proof is for the one-stage equilibrium, it is possible to prove uniqueness in a similar fashion for the other equilibria where there is a minimum effort in the second stage and three or more contestants. (Without a minimum bid the equilibrium is not unique for three or more contestants.)

Let $F_{i}, i=1, \ldots, n$, constitute an equilibrium profile of distribution functions of the contestants $i=1, \ldots, n$. The equilibrium profit of each contestant is strictly positive since bidding $m$ yields each contestant a strictly positive payoff by the assumption that $p(1-p)^{N-1} v>m$.

Using standard arguments it can be shown that the distribution functions must be atomless. Also, all contestants must have the same payoff in equilibrium. If contestant $i$ has a higher payoff than contestant $j$, contestant $j$ can bid at the top of the support of contestant $i$ and increase his payoff. Again, using standard arguments it can be shown that equal profits imply the distribution functions of contestants coincide over common supports. Each bid (strictly greater than $m$ ) in the support of a contestant $i$ must be in the support of at least one other contestant $j \neq i$ otherwise contestant $i$ could increase his payoff by bidding lower.

Let $z$ be the set of bids $x$ that belong to the support of at least two contestants. By standard arguments $z$ must be connected. Let $Z(x)$ be the distribution function of the two (or more) contestants which have $x$ in their support. By standard arguments $Z(x)$ must be strictly increasing on the common support.

If for some contestant, $i$, we have an $x^{\prime}$ such that $F_{i}\left(x^{\prime}\right)>Z\left(x^{\prime}\right)$, then $F_{i}$ cannot be increasing to the left of $x^{\prime}$, if it were, the point where it starts to increase must be in $z$ but $F_{i}$ cannot then increase to be strictly above $Z$ at this point t since $F_{i}$ is is atomless.

Also if there is an $x^{\prime}$ for which $F_{i}\left(x^{\prime}\right)<Z\left(x^{\prime}\right)$, then $F_{i}$ cannot be increasing to the right of $x^{\prime}$, since if it were, the point where it starts to increase must be in $z$ but $F_{i}$ cannot equal $Z$ at this point since $F_{i}$ is atomless.

Hence $F_{i}(x)$ equals $Z(x)$ for all $i$ and $x$ in $z$. This implies the unique equlibrium is given by the solution to Eq. 1 . 


\section{References}

Baye, M.R., Kovenock, D., De Vries, C.G.: The all-pay auction with complete information. Econ. Theory 8(2), 291-305 (1996). https://doi.org/10.1007/BF01211819

Chen, B., Jiang, X., Knyazev, D.: On disclosure policies in all-pay auctions with stochastic entry. J. Math. Econ. 70, 66-73 (2017)

Cohen, N., Maor, G., Sela, A.: Two-stage elimination contests with optimal head starts. Rev. Econ. Des. 22(3), 177-192 (2018)

Fu, Q., Jiao, Q., Lu, J.: On disclosure policy in contests with stochastic entry. Public Choice 148(3-4), 419-434 (2011)

Fu, Q., Jiao, Q., Lu, J.: Disclosure policy in a multi-prize all-pay auction with stochastic abilities. Econ. Lett. 125(3), 376-380 (2014)

Fu, Q., Jiao, Q., Lu, J.: Contests with endogenous entry. Int. J. Game Theory 44(2), 387-424 (2015)

Kaplan, T.R.: Communication of preferences in contests for contracts. Econ. Theory 51(2), 487-503 (2012). https://doi.org/10.1007/s00199-010-0525-2

Kaplan, T.R., Luski, I., Wettstein, D.: Innovative activity and sunk cost. Int. J. Ind. Organ. 21(8), 1111-1133 (2003)

McAfee, R.P., McMillan, J.: Auctions with a stochastic number of bidders. J. Econ. Theory 43(1), 1-19 (1987)

Segev, E., Sela, A.: Multi-stage sequential all-pay auctions. Eur. Econ. Rev. 70, 371-382 (2014a)

Segev, E., Sela, A.: Sequential all-pay auctions with head starts. Soc. Choice Welf. 43(4), 893-923 (2014b)

Sela, A.: Best-of-three all-pay auctions. Econ. Lett. 112(1), 67-70 (2011)

Sela, A.: Sequential two-prize contests. Econ. Theory 51(2), 383-395 (2012). https://doi.org/10.1007/ s00199-010-0547-9

Sela, A.: Two-stage contests with effort-dependent values of winning. Rev. Econ. Des. 21(4), 253-272 (2017)

Serena, M.: Quality contests. Eur. J. Polit. Econ. 46, 15-25 (2017)

Serena, M.: Harnessing beliefs to optimally disclose contestants types. Econ. Theory (2021). https://doi. org/10.1007/s00199-021-01378-1 (forthcoming)

Sheremeta, R.M.: Experimental comparison of multi-stage and one-stage contests. Games Econ. Behav. 68(2), 731-747 (2010)

Siegel, R.: All-pay contests. Econometrica 77(1), 71-92 (2009)

Troncoso-Valverde, C.: Releasing information in private-value second-price auctions. Econ. Theory $\mathbf{6 5}(3)$, 781-817 (2018). https://doi.org/10.1007/s00199-017-1039-y

Publisher's Note Springer Nature remains neutral with regard to jurisdictional claims in published maps and institutional affiliations. 